\title{
Self-enrichment in globular clusters
}

\section{An analytic approach}

\author{
S. Recchi ${ }^{1,2,3}$ and I. J. Danziger ${ }^{3}$ \\ 1 University Observatory of Vienna, Türkenschanzstrasse 17, 1180 Vienna, Austria \\ e-mail: recchi@astro.univie.ac.at \\ 2 Institut für Theoretische Physik und Astrophysik, Kiel University, Olshausenstrasse 40, 24098 Kiel, Germany \\ 3 INAF, Osservatorio Astronomico di Trieste, via G.B. Tiepolo, 11, 34131 Trieste, Italy \\ e-mail: danziger@ts.astro.it
}

Received 28 October 2004 / Accepted 21 February 2005

\begin{abstract}
By means of analytical calculations, we explore the self-enrichment scenario for Globular Cluster formation. According to this scenario, an initial burst of star formation occurs inside the core radius of the initial gaseous distribution. The outward-propagating shock wave sweeps up a shell in which gravitational instabilities may arise, leading to the formation of a second, metal-enriched, population of stars. We find a minimum mass of the proto-globular cluster of the order of $10^{6} M_{\odot}$. We also find that the observed spread in the magnitude-metallicity relation can be explained assuming cluster-to-cluster variations of some parameters like the thermalization efficiency, the mixing efficiency and the Initial Mass Function, as well as variations of the external pressure.
\end{abstract}

Key words. Galaxy: evolution - globular clusters: general - hydrodynamics

\section{Introduction}

Globular Clusters (GCs) are among the oldest objects in the Galaxy and thus are fossil records of its formation. Their age is comparable with the age of halo stars but, quite surprisingly, the lowest metallicity found in GCs $([\mathrm{Fe} / \mathrm{H}] \lesssim-2.5)$ is more than two orders of magnitude larger than the iron abundance of the most metal-poor halo stars (e.g. the recently discovered star HE 0107-5240 has a metallicity $[\mathrm{Fe} / \mathrm{H}]=-5.3$; Christlieb et al. 2004). This could be only a statistical effect, since GCs make up only $\sim 1 \%$ of the stars in the halo of the Galaxy. Moreover, the metallicity distribution of the halo stars and of the GCs peak at the same value (Ashman \& Zepf 1998). This could mean that the halo stars are the debris of the tidal disruption of cluster-like objects. Observations of the distribution of a wide sample of stars detected in the Sloan Digital Ski Survey (Newberg et al. 2002) seems to support this picture.

More detailed analyses of the metallicity distribution of halo stars and GCs (Laird et al. 1993; Carney et al. 1996) indicate that there is a statistically significant difference between the two metallicity distributions that cannot be explained by the lower numbers of GCs. Moreover, field halo stars are almost all $\mathrm{CN}$-weak, whereas some GCs show a bimodal CN distribution, with some stars having high $\mathrm{CN}$ abundances (Norris \& Smith 1981). This may indicate a difference in the formation process of these two classes of objects.
In many GCs the abundances of elements from $\mathrm{C}$ to Al show complex patterns and star-to-star abundance variations, such as the well-known $\mathrm{O}-\mathrm{Na}$ and $\mathrm{Mg}$-Al anticorrelations (Gratton et al. 2001; Gonzalez \& Wallerstein 1998). A few GCs: $\omega$ Centauri (Freeman \& Rodgers 1975; Smith et al. 2000; Pancino et al. 2002), M 22 (Norris \& Freeman 1983; Lehnert et al. 1991) and maybe M 92 (Langer et al. 1998) show a significant spread even in iron-peak abundances, with signatures of a metallicity gradient. Pancino et al. (2002) found also in $\omega$ Centauri that $[\mathrm{Fe} / \mathrm{H}]$ correlates with $[\mathrm{Cu} / \mathrm{Fe}]$, whereas the $[\mathrm{Si} / \mathrm{Fe}]$ vs. $[\mathrm{Fe} / \mathrm{H}]$ plot shows a plateau until $[\mathrm{Fe} / \mathrm{H}] \sim-1$ and then an anticorrelation. These patterns are characteristic of iron enrichment from SNeIa (Matteucci et al. 1993).

The range of metallicities in GCs is similar to that found in Local Group Dwarf Spheroidals (DSphs) and even in this case no galaxies with $[\mathrm{Fe} / \mathrm{H}] \lesssim-2.2$ are found. This may indicate a common origin of these two classes of objects, or at least a similar enrichment scenario.

In order to explain the lack of metal-poor GCs, two processes have been invoked: pre-enrichment from PopIII stars (Beasley et al. 2003) and the self-enrichment scenario, the second being the only one able to explain abundance variations observed in some GCs. The idea behind this is that, if a protoglobular cluster (PGC) is massive enough, it may retain the heavy elements produced by the first supernovae exploding after the collapse of the cloud. These metals may mix with the 


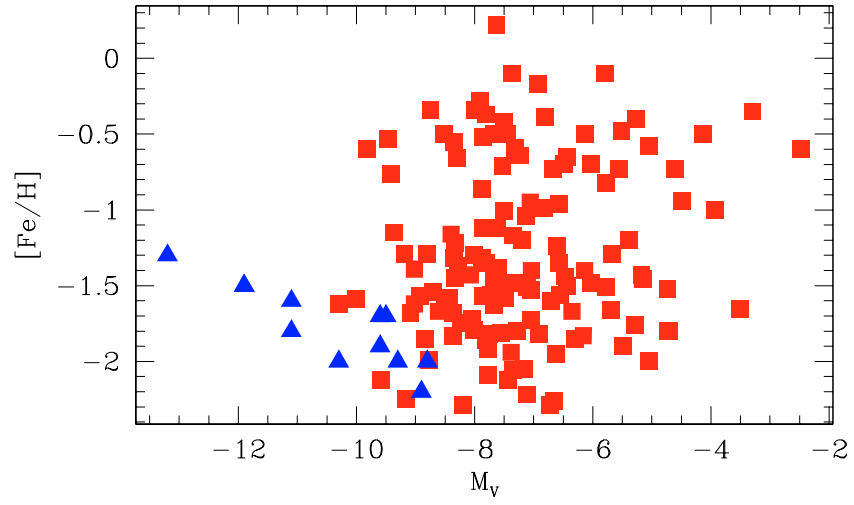

Fig. 1. Plot of the total visual magnitude $M_{V}$ vs. metallicity [Fe/H] for GCs of the Milky Way (squares) and Local Group DSphs (triangles). Data are from the Harris Catalogue (GCs) and from Mateo (1998) (DSphs).

surrounding, unpolluted medium and any following episodes of star formation arise from a metal-enriched gas. The pioneers in this kind of study have been Dopita \& Smith (1986) and Cayrel (1986), but many other authors have studied the subject (Morgan \& Lake 1989; Ikuta \& Arimoto 2000; Smith 2000 among others). In particular, Shustov \& Wiebe (2000; hereafter SW00) and Parmentier et al. (1999; hereafter P99) addressed the question of how massive a PGC should be in order to retain the products of at least one supernova (SN) and how many $\mathrm{SNe}$ can a PGC sustain before being disrupted as a result of the injected energy. P99 tried also to compare the results of their model with the magnitude-metallicity relation in a particular sample of Milky Way GCs (namely the older ones).

This is the first of a series of three papers dealing with analytical and numerical calculation of the self-enrichment process in GCs. In this paper, after carefully studying the available data about GCs and Local Group DSphs (Sect. 2), we perform an analytical study of the evolution of a PGC, taking into consideration the possibility of triggered star formation (Sect. 3). Results are presented and discussed in Sect. 4. Finally, we draw our conclusions in Sect. 5. In the forthcoming papers, we will analyze 1-D (Paper II) and 2-D (Paper III) chemodynamical simulations of the evolution of a PGC.

\section{On the mass dependence in the metallicity distribution of Milky Way GCs}

The most obvious reference for any study of Milky Way GCs is the Harris Catalogue (http://physun.physics. mcmaster.ca/Globular.html; see also Harris 1996). The metallicity-luminosity diagram for the whole galactic globular cluster system is shown in Fig. 1. We include also in the same plot the metallicity-luminosity relation for Local Group DSphs, taken from the Mateo (1998) review.

This plot does not show any obvious correlation among GCs, whereas the DSph system exhibits a well defined and well known trend, such that the faintest DSphs are also the most metal-poor. The classical explanation of this correlation is that the effect of galactic winds decreases with the increasing depth of the galactic potential well (Larson 1974;

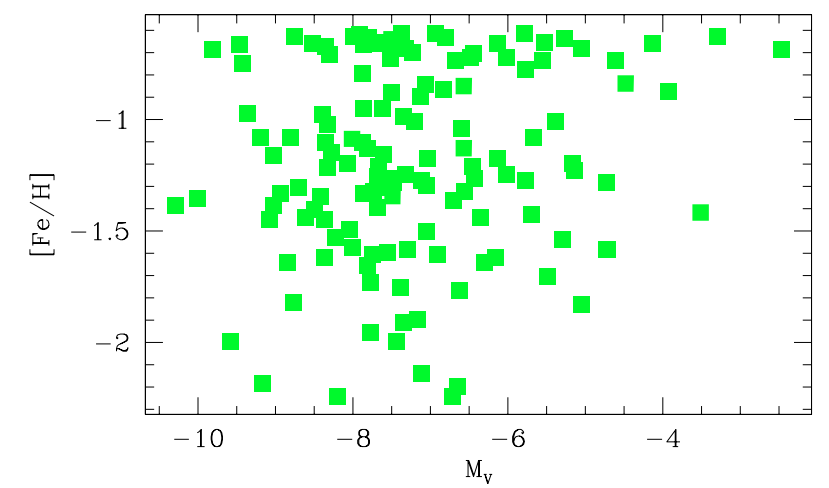

Fig. 2. Plot of the total visual magnitude $M_{V}$ vs. metallicity [Fe/H] for GCs of the Milky Way, by means of the calibration proposed by Carretta \& Gratton (1997).

Dekel \& Silk 1986). This mechanism cannot easily be extended to GCs, mostly because the DSphs show a very large mass-tolight ratio, presumably a hint of dominant dark matter halos, whereas GCs show very little evidence of dark matter.

There have been several attempts in the literature to calibrate the integrated light of GCs in order to recover the metallicity (Danziger 1973; Zinn 1980). The Harris catalogue is based on the Zinn \& West (1984) calibration, but this is not the only one and care is needed before drawing conclusions from the inspection of the metallicity-luminosity plot. In particular, Carretta \& Gratton (1997) proposed a revised version of the Zinn \& West (1984) calibration. This new calibration changes the resulting metallicity in particular in the range of high abundances. The metallicity-luminosity relation with this calibration is shown in Fig. 2.

It has been suggested that the mass loss due to stripping with passage through the disk of the Galaxy might dominate the mass budget of GCs. This could be the reason why there is such an enormous spread in this relation. One may suppose that the galaxies with large $|b|$, thus at large distance from the disk of the Galaxy, may have suffered less stripping. For this reason, we select the GCs with $|b|>20$ and plot in Fig. 3 the metallicity-luminosity relation for this selected sample of GCs, with both the Zinn \& West (1984) calibration (filled squares) and the Carretta \& Gratton (1997) one (open squares).

Although Fig. 3 shows a reduced spread compared to Figs. 1 and 2, an obvious correlation still cannot be detected, suggesting that, besides external effects, internal properties of the GCs should also be invoked in order to justify this enormous spread.

For a reduced sample of GCs, mass measurements are available. In this case, we do not have the problem to calibrate the metallicity index, but the determination of the mass of GCs is very uncertain and model-dependent (Pryor \& Meylan 1993). We adopt the same selection criterion introduced for Fig. 3 (i.e. selection of GCs at large galactic latitudes) and we plot in Fig. 4 the GC masses as a function of the metallicity for the GCs studied by Pryor \& Meylan (1993). Also in this case, no obvious trend can be identified, unless we exclude from the plot the two most massive GCs. In this case we can see that the metallicity slightly increases with mass. 


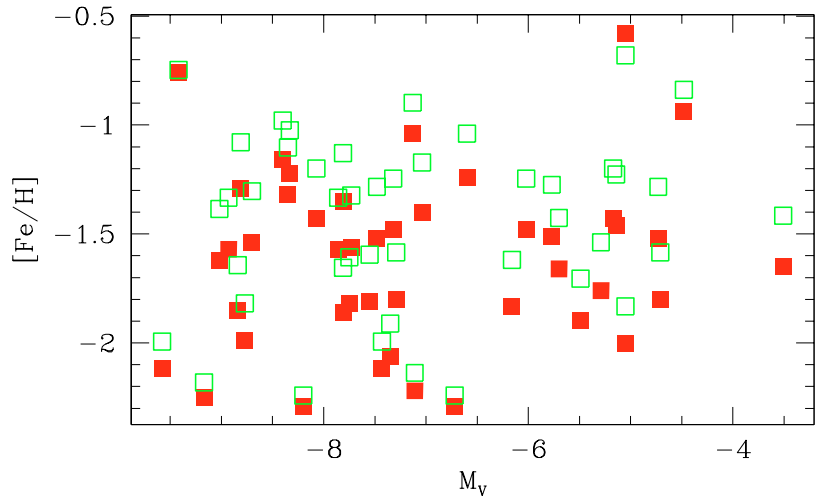

Fig. 3. Plot of the total visual magnitude $M_{V}$ vs. metallicity $[\mathrm{Fe} / \mathrm{H}]$ for the GCs of the Milky Way with $|b|>20$. The filled squares are relative to the Zinn \& West (1984) calibration, the open squares to the Carretta \& Gratton (1997) one.

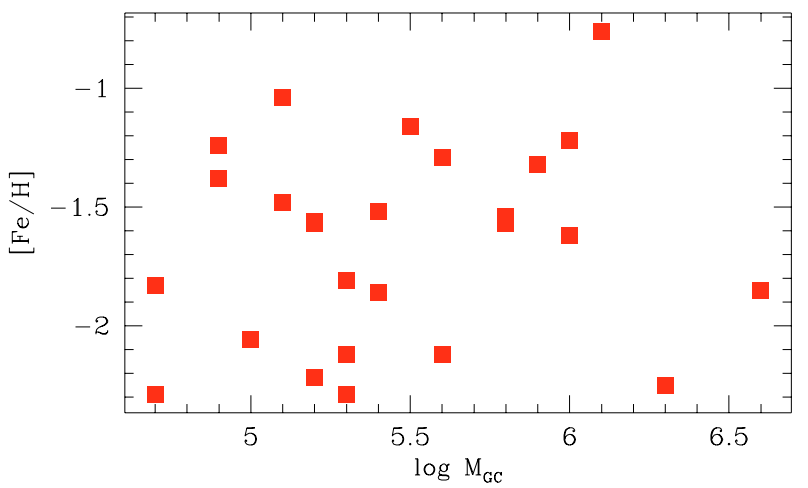

Fig. 4. Plot of the total GC mass $\log M_{\mathrm{GC}}$ vs. metallicity $[\mathrm{Fe} / \mathrm{H}]$ for the GCs of the Milky Way with $|b|>20$. Data are taken from Pryor \& Meylan (1993).

\section{An analytic approach}

\subsection{Set-up of the model}

Let us assume an isothermal sphere $(P \sim \rho)$ in hydrostatic equilibrium. The hydrostatic equilibrium, in spherical symmetry, is expressed by the formula $\frac{\mathrm{d} P}{\mathrm{~d} r}=-\frac{G M(r) \rho(r)}{r^{2}}$, whereas the conservation of mass implies that $\frac{\mathrm{d} M}{\mathrm{~d} r}=4 \pi \rho(r) r^{2}$. Combining these two equations, we obtain the fundamental equation of equilibrium:

$\frac{1}{r^{2}} \frac{\mathrm{d}}{\mathrm{d} r}\left(\frac{r^{2}}{\rho} \frac{\mathrm{d} P}{\mathrm{~d} r}\right)=-4 \pi G \rho(r)$.

We can modify this equation, introducing two dimensionless variables

$\left\{\begin{array}{l}\omega=-\ln \rho / \rho_{0} \\ \xi=A r, \quad A^{2}=\frac{4 \pi G \rho_{0}}{c_{\mathrm{s}}^{2}}\end{array}\right.$

where $c_{\mathrm{s}}$ is the sound speed, obtaining:

$\frac{\mathrm{d}^{2} \omega}{\mathrm{d} \xi^{2}}+\frac{2}{\xi} \frac{\mathrm{d} \omega}{\mathrm{d} \xi}=\mathrm{e}^{-\omega}$

This is the classical Lane-Emden equation for an isothermal sphere and cannot be solved analytically. With a simple,

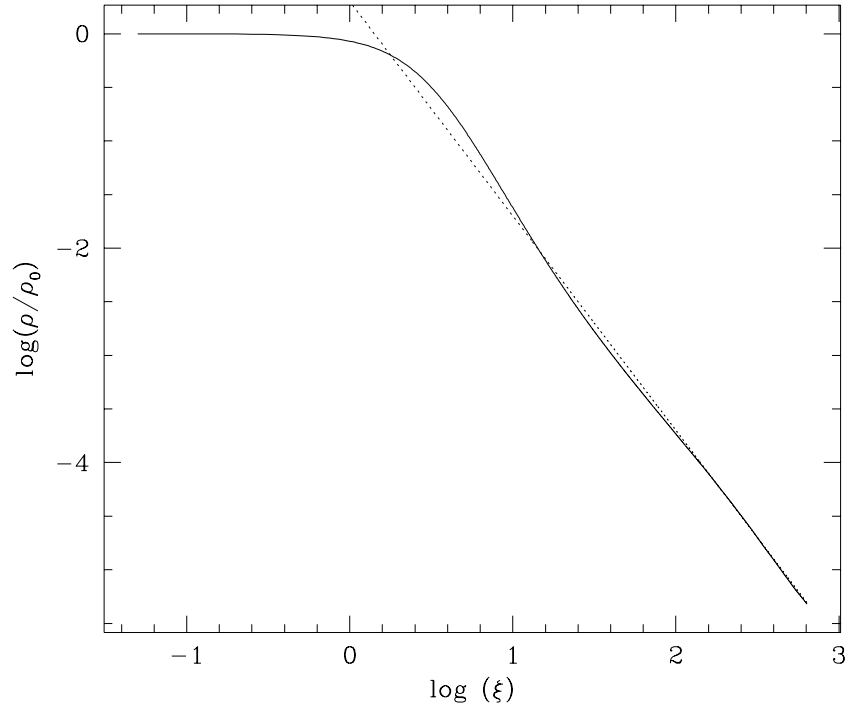

Fig. 5. Normalized density profile in an isothermal sphere. Solid line: solution of the numerical integration; dotted line: asymptotic trend at large $\xi$.

second-order numerical integration, we obtain the density profile plotted in Fig. 5. Also plotted (dotted line) is the asymptotic trend at large $\xi\left(\rho \sim \rho_{0} \cdot \xi^{-2}\right)$

This density profile can be well approximated by a King profile:

$\rho(r)= \begin{cases}\rho_{0} & \text { if } r<r_{\mathrm{c}} \\ \rho_{0} \frac{r_{\mathrm{c}}{ }^{2}}{r^{2}} & \text { if } r \geq r_{\mathrm{c}},\end{cases}$

where $r_{\mathrm{c}}$ is the core radius and the profile is truncated at a tidal radius $R$. The concentration parameter $c=\log \left(R / r_{\mathrm{c}}\right)$ is $\sim 1$ for most of metal-poor GCs (see Harris Catalogue), thus we can consider hereinafter $r_{\mathrm{c}}=0.1 \mathrm{R}$. We assume pressure equilibrium between the PGC and the external medium, thus, defining $P_{\mathrm{h}}$ as the external pressure, we obtain $\rho(R)=0.01 \rho_{0}=P_{\mathrm{h}} / c_{\mathrm{s}}{ }^{2}$. We use this expression to calculate the mass of the PGC as a function of $R$ and $P_{\mathrm{h}}$. Finally, this expression can be used to obtain the tidal radius $R$ as a function of the total PGC mass and the external pressure. The final expression is:

$R=\left[\frac{1}{2.8} \frac{3 k T}{4 \pi \mu m_{\mathrm{H}}}\right]^{1 / 3}\left(\frac{M}{P_{\mathrm{h}}}\right)^{1 / 3}$.

Assuming $\mu=1.3$ and $T=10^{4}$ (as we will do hereinafter), we obtain:

$R_{100}=1.385\left(\frac{M_{6}}{P_{4}}\right)^{1 / 3}$,

where $R_{100}$ is in units of $100 \mathrm{pc}, M_{6}$ in units of $10^{6} M_{\odot}$ and $P_{4}$ in units of $10^{4} k$. The dependence of $\rho_{0}$ with $P_{\mathrm{h}}$ is $\rho_{0,22}=2.17 P_{4}$, where $\rho_{0,22}$ is in units of $10^{-22} \mathrm{~g} \mathrm{~cm}^{-3}$.

\subsection{The collapse of the PGC}

Let us now assume that the sphere loses its equilibrium and starts collapsing. This assumption is justified by the fact that in 
Eq. (1) we neglected the cooling, thus the configuration should lose the equilibrium due to radiative losses. The cooling time in the inner core $\left(t_{\mathrm{c}} \simeq 3 k T /(2 n \Lambda(T))\right.$, where $\Lambda(T)$ is the cooling function) is approximatively $2 \times 10^{4} \mathrm{yr}$ with our assumptions. The collapse of the core will be much faster than the collapse of the external mantel. If we assume for simplicity the free fall time-scale of the mantel as $t_{\mathrm{ff}, \text { man }}=\sqrt{\left[3 \pi /\left(32 G \bar{\rho}_{\text {man }}\right)\right]}$, where $\bar{\rho}_{\text {man }}=3 \rho_{0} r_{\mathrm{c}}^{2}\left(R-r_{\mathrm{c}}\right) /\left(R^{3}-r_{\mathrm{c}}^{3}\right)$ is the average density of the external mantel, we obtain $t_{\mathrm{ff}, \mathrm{c}} / t_{\mathrm{ff}, \text { man }} \sim 0.16$; that is, if we assume for simplicity two separate collapse episodes, the mantel will not have time to loose its equilibrium configuration in the interval of time in which the core collapses. We can thus assume that a fraction of the core collapses and forms stars in an instantaneous burst of star formation, while the external mantel keeps its starting density profile.

The efficiency of star formation in the core (namely the fraction of core mass turned into stars) is assumed to be 0.3 , consistent with the star formation efficiencies obtained in the cores of dense clouds by Bate et al. (2003).

The mass of stars in this first, almost metal-free, episode of star formation is thus:

$M_{*, 6}=1.071 \times 10^{-2} M_{6}$.

The number of SNeII is given by: $N_{\mathrm{SNII}}=\int_{8}^{100} A m^{-x} \mathrm{~d} m$, where $A$ is calculated from $M_{*}=\int_{m_{1}}^{100} A m^{1-x} \mathrm{~d} m$. A Salpeter $(x=$ 2.35 ) index can be taken as a good approximation to the IMF slope in clusters (Kroupa 2002). $m_{1}$ is the lower cut-off of the IMF. We define $g\left(m_{1}\right)=m_{1}^{-0.35}-100^{-0.35}$ and we take $m_{1}$ as a free parameter. We obtain:

$N_{\mathrm{SNII}}=162.1 \frac{M_{6}}{g\left(m_{1}\right)}$.

For a reasonable choice of $m_{1}$ (i.e. $\left.m_{1}=1\right)$ this gives $N_{\mathrm{SNII}} \simeq$ $200 M_{6}$, thought by P99 to be the critical number of SNe sustainable against PGC disruption. Assuming the rate of SNeII constant in time, we can calculate the luminosity of the burst. This is given by: $L=\eta E_{\mathrm{SNII}} N_{\mathrm{SNII}} / \Delta t$, where $\eta$ is the fraction of the mechanical energy of the explosion which goes into thermal energy of the medium (the so-called thermalization efficiency), $E_{\text {SNII }}$ is the energy of a single SNII (assumed to be $10^{51} \mathrm{erg}$ ) and $\Delta t \sim 30 \mathrm{Myr}$ is the interval of time in which SNeII explode. The thermalization efficiency can be evaluated assuming the evolution of a SNR in a uniform medium. We can calculate the time at which the expansion velocity of the SNR becomes equal to the local sound speed, the kinetic energy of the shell at this time, and divide this energy by the blast wave energy (see Cioffi et al. 1988; Bradamante et al. 1998; Recchi et al. 2001, for more details). We obtain:

$\eta \simeq 0.02 n_{0}^{-54 / 49} \xi^{-15 / 98} c_{0,6}^{5 / 7}$,

where $\xi$ is the metallicity (in units of $Z_{\odot}$ ) and $c_{0,6}$ is the local sound speed (in units of $10^{6} \mathrm{~cm} \mathrm{~s}^{-1}$ ). Assuming $\xi=10^{-4}$ (typical of the most metal-poor halo stars), we obtain:

$\eta=4.36 \times 10^{-4} P_{4}^{-54 / 49}$.

It is worth mentioning that the determination of $\eta$ in this way is too simplistic and many other physical processes contribute to its determination (see Melioli \& de Gouveia Dal Pino 2004). Therefore we will keep $\eta$ in this paper as a free parameter, bearing in mind that, at least in the initial stages of the evolution of the system, its value might be very low. The luminosity of the burst (in units of $10^{38} \mathrm{erg} \mathrm{s}^{-1}$ ) is thus given by:

$L_{38}=1.712 M_{6}\left(\frac{\eta}{g\left(m_{1}\right)}\right)$.

We assume that mass and energy of this first generation of stars are released in a spherical volume of radius $r_{\mathrm{SF}} \ll R$ and we keep the $r^{-2}$ gas density profile outside it.

\subsection{Slow winds or fast winds?}

The evolution of wind-blown bubbles and superbubbles has been carefully analyzed by Koo \& McKee (1992a,b). Since the results of these authors will be extensively used in the paper, we recall here briefly a few results about bubble expansion. The freely expanding wind produced by the starburst interacts supersonically with the unperturbed ISM, creating a classical bubble structure (Weaver et al. 1977), in which two shocks are present. The external one ("ambient shock") propagates through the ISM and creates an expanding cold and dense shell. A second shock ("wind shock") propagates inwards, thermalizing the impinging wind and creating the hot, rarefied gas of the bubble interior. The shocked ambient medium and the shocked wind are separated by a contact discontinuity. Initially, the wind density is large and the velocity of the wind shock is small, therefore the wind shock is radiative. At later stages, the wind density decreases and the wind shock velocity increases, therefore the wind shock is expected to become adiabatic. If the energy input is powerful enough, this transition could occur in an early stage of the bubble expansion, when the wind is still freely expanding. In this case, the radiative cooling of the cavity is dynamically unimportant. Koo \& McKee (1992a) call these kinds of winds fast winds. The slow winds are instead the ones in which the transition from a radiative to an adiabatic wind shock occurs later, making the cooling of the bubble dynamically relevant.

Considering a generic power-law density distribution of the ambient medium $\rho(r)=\rho_{01} r^{-k_{\rho}}$ and a generic rate of energy injection $L_{\text {in }}=\mathcal{L} t^{\eta_{\text {in }}-1}$, Koo \& McKee $(1992 b)$ were able to calculate a critical velocity, namely the velocity which divides the two types of winds. This expression is given by:

$v_{\text {cr }}=\left[\left(\frac{\mathcal{L}}{2 \pi \eta_{\text {in }}}\right)^{1-k_{\rho}}\left(\frac{3 \rho_{01}}{3-k_{\rho}}\right)^{2-\eta_{\text {in }}} \frac{1}{C_{1}^{3-k_{\rho}-\eta_{\text {in }}}}\right]^{\alpha_{k, \eta}}$,

where $\alpha_{k, \eta}=1 /\left[14-6 k_{\rho}-\left(3+k_{\rho}\right) \eta_{\text {in }}\right]$ and $C_{1}=6 \times$ $10^{-35} \mathrm{~g} \mathrm{~cm}^{-6} \mathrm{~s}^{4}$.

Assuming a $r^{-2}$ profile and a constant injection rate (i.e. $\left.\eta_{\text {in }}=1\right)$ Eq. (12) becomes:

$v_{\mathrm{cr}}=\left(\frac{\mathcal{L}}{6 \pi \rho_{0} r_{\mathrm{c}}^{2}}\right)^{1 / 3}$.

By substituting our parameters, we obtain:

$v_{\mathrm{cr}, 8}=2.84 \times 10^{-2}\left(\frac{M_{6}}{P_{4}}\right)^{1 / 9}\left(\frac{\eta}{g\left(m_{1}\right)}\right)^{1 / 3}$. 
Table 1. Ejecta fraction and mass in metals.

\begin{tabular}{ccc}
\hline \hline$M_{\text {in }}$ & $f_{\text {ej }}$ & $m_{z}$ \\
\hline 12 & 0.90 & 0.48 \\
13 & 0.89 & 0.50 \\
15 & 0.90 & 0.73 \\
18 & 0.92 & 1.58 \\
20 & 0.90 & 2.34 \\
22 & 0.91 & 2.74 \\
25 & 0.93 & 4.06 \\
30 & 0.94 & 6.01 \\
35 & 0.92 & 7.18 \\
40 & 0.90 & 8.22 \\
\hline
\end{tabular}

Total ejected fraction $\left(f_{\mathrm{ej}}=1-M_{\text {rem }} / M_{\mathrm{in}}\right)$ and mass of metals $m_{z}$ as a function of initial mass. Data from Woosley \& Weaver $1995(Z=$ $10^{-4} Z_{\odot}$; case B).

An approximate value of the velocity of the ejecta from SNeII is given by $v_{\mathrm{ej}}=\sqrt{2 E_{\mathrm{SNII}} / M_{\mathrm{ej}, \mathrm{SNII}}}$, where $E_{\mathrm{SNII}}=\eta \cdot N_{\mathrm{SNII}} \times$ $10^{51} \mathrm{erg}$ is the total energy released by SNeII, whereas $M_{\mathrm{ej}, \mathrm{SNII}}$ is the total mass restored by these $\mathrm{SNe}$. This mass is a fraction $f_{\mathrm{ej}}$ of the total mass in stars in the interval $[8,100] M_{\odot}$. Assuming a Salpeter IMF, the mass of stars in this interval is $0.283 / g\left(m_{1}\right)$ times the total mass of stars, whereas $f_{\mathrm{ej}}$ can be evaluated from the tables of Woosley \& Weaver (1995). Assuming an abundance of the PGC of $10^{-4} Z_{\odot}, f_{\mathrm{ej}} \sim 0.9$, with almost no dependence on the initial mass (see Table 1), we thus obtain:

$v_{\mathrm{ej}, 8}=2.44 \sqrt{\eta}$,

where $v_{\mathrm{ej}, 8}$ is in units of $10^{8} \mathrm{~cm} \mathrm{~s}^{-1}$.

The ratio $v_{\mathrm{ej}} / v_{\mathrm{cr}}$ is given by:

$\frac{v_{\text {ej }}}{v_{\text {cr }}}=85.9\left(\frac{M_{6}}{P_{4}}\right)^{-1 / 9} \eta^{1 / 6} g\left(m_{1}\right)^{1 / 3}$,

which is very large and varies very little with the parameter space. Even assuming the (very low) thermalization efficiency found in Eq. (10), assuming $m_{1}=1$ and neglecting the very weak dependence on $M_{6}$ and $P_{4}$, we obtain $v_{\mathrm{ej}} / v_{\mathrm{cr}} \simeq 20$. We can thus safely state that the winds occurring in PGC are fast winds.

\subsection{The evolution of the superbubble}

Koo \& McKee (1992a) defined the fiducial radius $R_{\mathrm{f}}$ as the radius at which the wind density equals the ambient density. For a general power-law density distribution and a general rate of energy injection, they obtained:

$R_{\mathrm{f}}=\left[\frac{\left(3-k_{\rho}\right) \mathcal{L}}{6 \pi \eta_{\text {in }} \rho_{01} v_{\text {in }}^{2+\eta_{\text {in }}}}\right]^{\frac{1}{3-k_{\rho}-\eta_{\text {in }}}}$.

Koo \& McKee (1992b) were able to derive the expansion law of the bubble under various initial conditions and in different stages of the bubble evolution, as a function of $R_{\mathrm{f}}$. Indeed, ours is a special case, since, for $k_{\rho}=2$ and $\eta_{\text {in }}=1$ this normalization breaks down and the fiducial radius approaches zero. This special case is called constant velocity bubble and, physically, this breakdown corresponds to the fact that the ratio between swept-up mass and ejected mass is constant.

The ambient medium always dominates these kinds of winds and the bubble always stays radiative. The superbubble expands with a constant velocity $v_{\mathrm{b}}=v_{\mathrm{ej}} I /(1+I)$, where $I=\sqrt{\mathcal{L} /\left(2 \pi \rho_{0} r_{\mathrm{c}}^{2} v_{\mathrm{ej}}^{3}\right)}=\sqrt{3} \cdot\left(v_{\mathrm{cr}} / v_{\mathrm{ej}}\right)^{3 / 2}$. With our parameters:

$v_{b, 6} \simeq 0.532\left(\frac{M_{6}}{P_{4}}\right)^{1 / 6} \eta^{1 / 4} g\left(m_{1}\right)^{-1 / 2}$

(where $v_{b, 6}$ is in units of $10^{6} \mathrm{~cm} \mathrm{~s}^{-1}$ ) and consequently the superbubble expands with a law:

$R_{s, 100}=5.44 \times 10^{-2}\left(\frac{M_{6}}{P_{4}}\right)^{1 / 6} \eta^{1 / 4} g\left(m_{1}\right)^{-1 / 2} t_{6}$,

with $t_{6}$ in units of $10^{6} \mathrm{yr}$. Since the burst originates in a spherical region of radius $r_{\mathrm{c}}, R_{\mathrm{s}}$ should be considered as the difference between the shock radius and $r_{\mathrm{c}}$. Note that, if the bubble evolves into a partially radiative bubble (i.e. a bubble in which most of the shocked wind has cooled down, but most of the bubble volume is filled with the most recently shocked and still hot portion of the wind), the velocity would be no more constant, but increasing with $t^{1 / 3}$. However, such an accelerating bubble would produce Rayleigh-Taylor instabilities with the overlying gas, resulting in a larger cooling of the shocked wind, preventing the onset of the partially radiative bubble (see Koo $\&$ McKee 1992b). Therefore, the assumption of a constantvelocity bubble is reliable. The time needed to reach the tidal radius $R$ is:

$t_{\text {tid, } 6}=22.9\left(\frac{M_{6}}{P_{4}}\right)^{1 / 6} \eta^{-1 / 4} g\left(m_{1}\right)^{1 / 2}$,

which is of the order of the lifetime of a $8 M_{\odot}$ star. It implies that the assumption of a constant luminosity is reliable.

The swept-up mass as a function of time is given by:

$M_{\text {sw, } 6}=4.2 \times 10^{-2} M_{6}^{5 / 6} P_{4}^{1 / 6} \eta^{1 / 4} g\left(m_{1}\right)^{-1 / 2} t_{6}$.

It is easy to check that the swept-up mass at $t_{\text {tid }}$ is similar to the initial mass; the difference being the mass in stars.

The mass $m_{Z}$ of metals ejected in the ISM by a SN whose progenitor mass is $M_{\text {in }}$ is shown in Table 1. This dependence can be approximated by:

$m_{Z}\left(M_{\text {in }}\right)=0.015\left(M_{\text {in }}-8\right)^{2}-\frac{2}{3} \times 10^{-5}\left(M_{\text {in }}-8\right)^{4}$,

(see Fig. 6). This approximation is valid until $M_{\text {in }}=40 M_{\odot}$. We will assume a constant $m_{Z}$ above $40 M_{\odot}$. The total mass of metals released into the ISM as a function of time can be expressed as:

$$
\begin{aligned}
M_{Z}(t) & =\frac{0.35}{g\left(m_{1}\right)} M_{*} \int_{100}^{m(t)} M_{\mathrm{in}}^{-2.35} m_{Z}\left(M_{\mathrm{in}}\right) \mathrm{d} M_{\mathrm{in}} \\
& =\frac{0.35}{g\left(m_{1}\right)} M_{*} \Delta_{Z}(t) .
\end{aligned}
$$




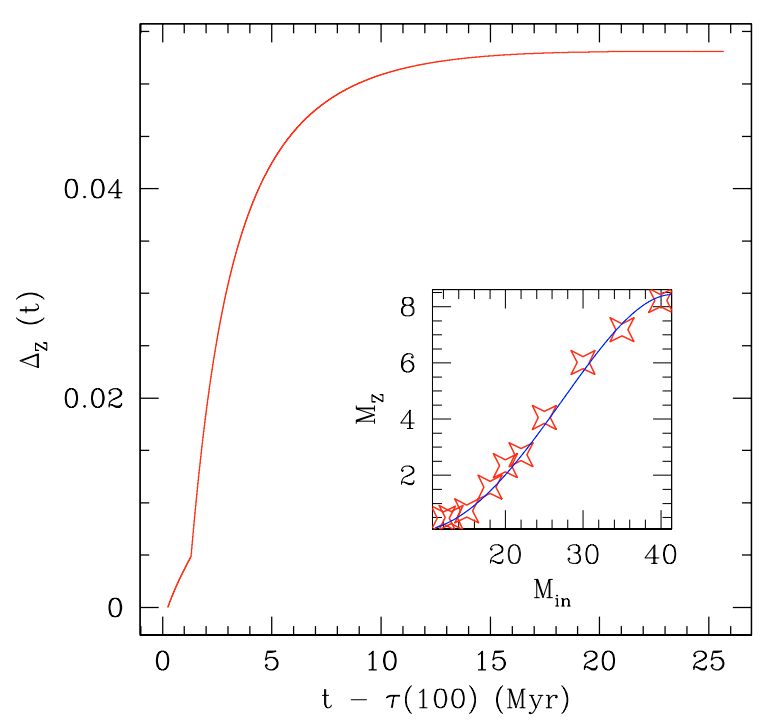

Fig. 6. Normalized metal production $\Delta_{Z}$ as a function of time. $\tau(100)$ is the lifetime of a $100 M_{\odot}$ star. In the inner plot is shown the mass $m_{Z}$ of heavy elements ejected by a SN of initial mass $M_{\text {in }}$ and our approximation to this trend.

Table 2. Normalized metal production $\Delta_{Z}$ as a function of time.

\begin{tabular}{cc}
\hline \hline$t-\tau(100)(\mathrm{Myr})$ & $\Delta_{Z}(t) * 100$. \\
\hline 1. & 0.367 \\
2. & 1.875 \\
3. & 3.089 \\
4. & 3.797 \\
5. & 4.245 \\
6. & 4.544 \\
7. & 4.572 \\
8. & 4.900 \\
9. & 5.008 \\
10. & 5.087 \\
11. & 5.147 \\
12. & 5.191 \\
13. & 5.224 \\
14. & 5.249 \\
15. & 5.267 \\
16. & 5.281 \\
17. & 5.291 \\
18. & 5.298 \\
19. & 5.303 \\
20. & 5.307 \\
21. & 5.309 \\
22. & 5.309 \\
23. & 5.309 \\
24. & 5.309 \\
25. & 5.309 \\
\hline & \\
\hline
\end{tabular}

Normalized metal production as a function of time (see Eq. (23) for the definition of $\left.\Delta_{Z}(t)\right)$.

This integral cannot be evaluated analytically. We solve it numerically. We assume a simple law for the stellar lifetimes: $t(m)=1200 m^{-1.85}-3 \mathrm{Myr}$ (Padovani \& Matteucci 1993). The resulting $\Delta_{Z}(t)$ is shown in Fig. 6 and the values every Myr are tabulated in Table 2 .

\subsection{Triggered star formation in the shell}

The expansion of a supershell can trigger star formation in the shell itself if the onset of gravitational instabilities in the fragments occurs when the shell is still bound to the PGC potential. Moreover, we must require that the time-scale for the onset of these gravitational instabilities is shorter than the time-scale needed to reach the tidal radius of the PGC. In order to calculate the time-scale needed for the onset of gravitational instabilities, we follow an approach similar to the one of McCray \& Kafatos (1987), namely we consider a small circular disk with radius $\xi \ll r$ on the surface of the expanding spherical shell, which expands in a $r^{-2}$ density profile with a constant velocity $v_{\mathrm{b}}$. We indicate with $\theta \simeq \xi / r$ the halfangle subtended by the disk. The thermal, kinetic and gravitational energy of the disk are $m c_{\mathrm{s}}^{2}, \frac{1}{2} m\left(\frac{\mathrm{d} \xi}{\mathrm{d} t}\right)^{2},-\frac{2}{3} G \frac{m^{2}}{\xi}$, respectively. The mass of the disk is $m(r, \theta)=\pi \theta^{2} \rho_{0} r_{\mathrm{c}}^{2}\left(r-r_{\mathrm{c}}\right)$, thus we obtain: $E_{K}=\frac{1}{2} \pi \theta^{4} v_{\mathrm{b}}^{2} \rho_{0} r_{\mathrm{c}}^{2}\left(r-r_{\mathrm{c}}\right) ; E_{\mathrm{Th}}=\pi \theta^{2} \rho_{0} r_{\mathrm{c}}^{2}\left(r-r_{\mathrm{c}}\right) c_{\mathrm{s}}^{2}$; $E_{B}=-\frac{2}{3} G \pi^{2} \theta^{3} \rho_{0}^{2} r_{\mathrm{c}}^{4}\left(r-r_{\mathrm{c}}\right)^{2} / r$. The criterion for the onset of a gravitational instability is approximatively $E_{K}+E_{\mathrm{Th}}+E_{B}<0$, which transforms into:

$\frac{1}{2} \theta^{2} v_{\mathrm{b}}^{2}-\frac{2}{3} G \pi \theta \rho_{0} r_{\mathrm{c}}^{2} \frac{r-r_{\mathrm{c}}}{r}+c_{\mathrm{s}}^{2}<0$.

The $\Delta$ of this equation must be $>0$, otherwise the above expression is positive for any value of $\theta$. This transforms into the following condition:

$r>r_{\mathrm{c}}\left[1-\frac{3 c_{\mathrm{s}} v_{\mathrm{b}}}{\sqrt{2} G \pi \rho_{0} r_{\mathrm{c}}^{2}}\right]^{-1}$,

provided that $3 c_{\mathrm{s}} v_{\mathrm{b}}<\sqrt{2} G \pi \rho_{0} r_{\mathrm{c}}^{2}$.

This radius is reached at a time

$t_{*, 6}=\frac{2.55\left(\frac{M_{6}}{P_{4}}\right)^{1 / 6} \eta^{-1 / 4} g\left(m_{1}\right)^{1 / 2}}{1-11.07\left(M_{6} P_{4}\right)^{-1 / 2} \eta^{1 / 4} g\left(m_{1}\right)^{-1 / 2}}$.

The metallicity of this self-enriched population of stars is simply given by:

$Z=\gamma M_{Z}\left(t_{*}\right) / M_{\mathrm{sw}}\left(t_{*}\right)$,

where $\gamma$ is the mixing efficiency, namely the fraction of metals produced in the first generation of stars, able to mix with the surrounding cold shell in a time-scale shorter than the timescale needed for the onset of gravitational instabilities in the shell.

\section{Results}

\subsection{Constraints on the mass of the PGC}

The time needed for the onset of a gravitational instability able to generate the population of stars we observe nowadays in GCs $\left(t_{*, 6}\right)$ is given by Eq. (26). This time-scale has to be shorter than the time-scale needed to reach the tidal radius $\left(t_{\text {tid, } 6}\right.$, given by Eq. (20)), otherwise the energy released by the first generation of stars simply blows away all the gas in the PGC. By comparing these two time-scales, we obtain a condition for the mass of the PGC:

$M_{\mathrm{Min}, 1}>12.46 P_{4}^{-1 / 2} \eta^{1 / 4} g\left(m_{1}\right)^{-1 / 2}$. 


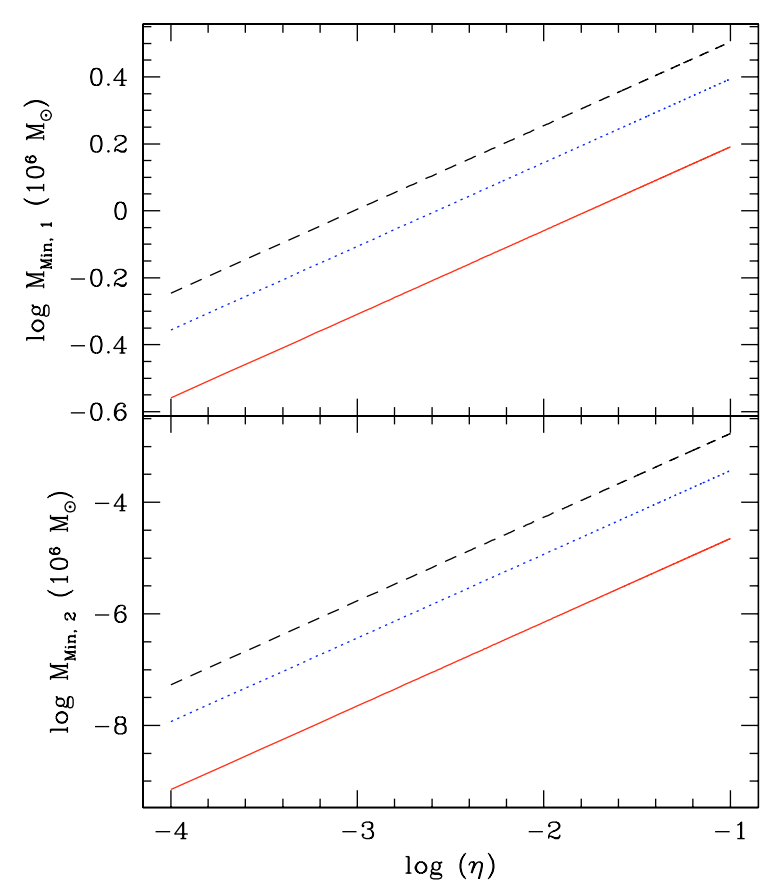

Fig. 7. Lower limits for the mass of the PGC calculated according to Eq. (28) (upper panel) and Eq. (30) (lower panel). The thermalization efficiency $\eta$ varies with continuity, whereas the lower IMF mass $m_{1}$ is equal to 0.1 (solid line), 1 (dotted line), 3 (dashed line).

We can obtain another constraint on $M_{6}$ by considering that, in the moment in which stars form in the shell, the shell has to be bound. It means that the potential energy of the shell should be larger than its kinetic energy, i.e. $\frac{1}{2} M_{\mathrm{sw}}\left(t_{*}\right) v_{\mathrm{b}}^{2}<\frac{1}{2} \frac{G M_{\mathrm{sw}}\left(t_{*}\right)^{2}}{R_{\mathrm{s}}\left(t_{*}\right)}$. In this way we can define a minimum shell mass:

$M_{\text {sh min, } 6}=\frac{9.11 \times 10^{-2}\left(\frac{M_{6}}{P_{4}}\right)^{2 / 3} \eta^{1 / 2} g\left(m_{1}\right)^{-1}}{1-11.07\left(M_{6} P_{4}\right)^{-1 / 2} \eta^{1 / 4} g\left(m_{1}\right)^{-1 / 2}}$.

The swept-up mass calculated by means of Eq. (21) has to be larger than $M_{\mathrm{sh} \text { min }}$ in order that the shell be bound. This translates into another condition for the mass of the PGC, namely:

$M_{\text {Min,2 }}>0.6 P_{4}^{-2} \eta^{3 / 2} g\left(m_{1}\right)^{-3}$.

As usual, all the masses (Eqs. (28)-(30)) are in units of $10^{6} M_{\odot}$.

The resulting dependence of the minimum mass of the PGC calculated according to Eq. (28) $\left(M_{\mathrm{Min} 1}\right)$ and Eq. (30) $\left(M_{\mathrm{Min}, 2}\right)$ is shown in Fig. 7 (as a function of $\eta$ ) and in Fig. 8 (as a function of $m_{1}$ ). For simplicity, the external pressure is kept constant in these plots and in the following sections. It is assumed to be $P_{4}=10$, in agreement with the pressure of the hot protogalactic background (Fall \& Rees 1985; Murray \& Lin 1992). We analyze the dependence of $[\mathrm{Fe} / \mathrm{H}]$ on $P_{4}$ in Sect. 4.2.4. These plots show that for any reasonable choice of parameters, the critical mass required in order to get the shell bound to the PGC potential well $\left(M_{\mathrm{Min}, 2}\right)$ is always extremely low. This is therefore not a severe constraint on the minimum mass of the PGC.

The constraint based on the time needed for the onset of a gravitational instability compared to the time required for the shock to reach the tidal radius (expressed by Eq. (28)) is more stringent and led us to consider only PGC with masses

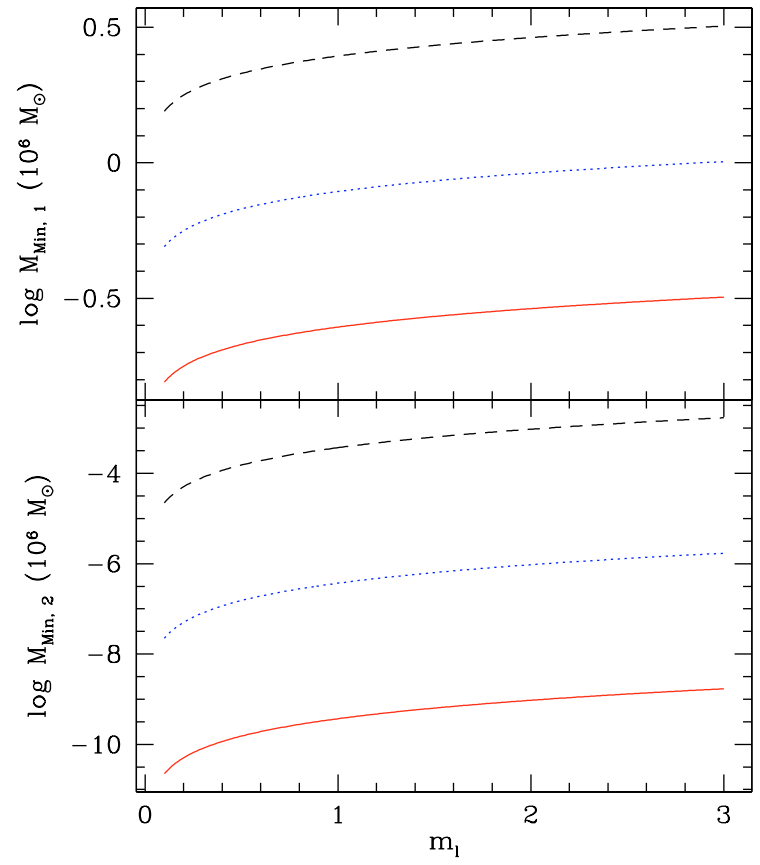

Fig. 8. As Fig. 6 but with varying lower IMF mass $m_{1}$. The thermalization efficiency $\eta$ is equal to $10^{-5}$ (solid line), $10^{-3}$ (dotted line), 0.1 (dashed line).

larger than $\sim 10^{6} M_{\odot}$. This is the same threshold mass found by SW00 and is also comparable with the threshold mass found by Brown et al. (1995), but is significantly larger than the one found by Morgan \& Lake (1989).

\subsection{The magnitude-metallicity relation in GCs}

A direct comparison between the results of this simplified model and the observations can be made as follows. We can assume the same efficiency of star formation adopted in Sect. 3.2, namely that $30 \%$ of the mass of the shell at the moment of the onset of the gravitational instability $\left(M_{\mathrm{sw}}\left(t_{*}\right)\right)$ transforms into stars. This is therefore the mass of our newly formed GC. This should be considered as a lower limit, since, after the formation of the second, polluted generation of stars, the GC is still rich in gas. Shocks created by the supernovae exploding in the shell can still compress the gas, leading to the formation of new stars.

To convert the mass in stars into visual magnitude, we assume a constant mass-to-light ratio $M / L_{V}=1.5$, typical for GC systems in the Milky Way (Harris 1996), M 31 (Djorgovski et al. 1997) and M 33 (Larsen et al. 2002). At this point the visual magnitude is simply calculated by means of the following formula:

$M_{V}=5.41-2.5 \cdot \log \left[0.2 \cdot M_{\mathrm{sw}}\left(t_{*}\right)\right]$.

Now we can directly compare the observed magnitudemetallicity relation with the results of our approximations. We take the sample of data already shown in Fig. 3, namely the GCs of the Milky Way with $|b|>20$, in order to avoid the effect of the mass loss due to stripping passing through the disk of the Galaxy. We explore the parameter space, varying the 


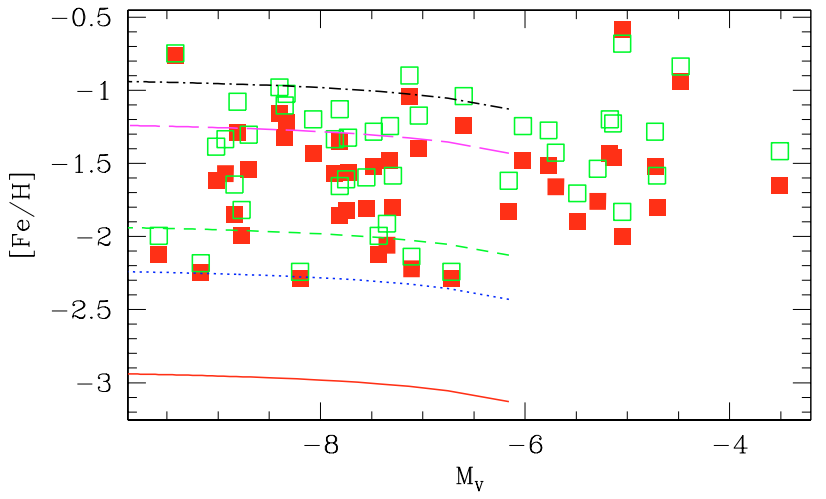

Fig. 9. Observed magnitude-metallicity relation for a selected sample of GCs (see Fig. 3) compared with model results. The mixing efficiency $\gamma$ is taken as 0.01 (solid line), 0.05 (dotted line), 0.1 (dashed line), 0.5 (long-dashed line) and 1 (dot-dashed line).

mixing efficiency $\gamma$, the thermalization efficiency $\eta$, the lower limit of the IMF distribution $m_{1}$ and the external pressure $P_{4}$.

\subsubsection{The magnitude-metallicity relation as a function of $\gamma$}

The mixing between the metals produced in the first generation of stars and the swept-up shell is a complex process, involving poorly known physics. In the idealized wind-blown bubble model, the shocked wind (hot, central region filled with metals) and the shocked ISM (the shell of unpolluted swept-up material) are separated by a contact discontinuity, thus in principle no flux of matter is possible between these two regions. Actually many physical processes can allow a mixing (mixing layers, turbulence, thermal instabilities, thermal conduction), but these processes are poorly constrained. We therefore decided to keep the mixing efficiency $\gamma$ as a free parameter, trying to find some constraint on its value through the comparison of our results with the observations.

The magnitude-metallicity relation as a function of $\gamma$ is shown in Fig. 9. In this set of models, the thermalization efficiency $\eta$ and the lower limit of the IMF distribution $m_{1}$ are set to be $10^{-4}$ and 1 , respectively. Although the value of the thermalization efficiency is rather low, it is fully consistent with the value found in Eq. (10). As we can see in this figure, the minimum required mixing efficiency is between 0.05 and 0.1 , whereas, in order to reach the most metal-rich clusters, a very large $\gamma$ is required. A non-negligible mixing between the hot cavity, filled with metals, and the shell, should therefore occur, but the magnitude of this process can be only poorly constrained due to the huge spread in the observed metallicities of GCs. Owing to the variety of processes contributing to the mixing of metals with the shell, the spread in metallicity can reflect, at least in part, a cluster-to-cluster variation of $\gamma$.

It is also worth noting that the evolution of the metallicity [Fe/H] as a function of $M_{V}$ is almost flat. This is in agreement with the fact that no evident trend is shown in the observed $M_{V}$ vs. $[\mathrm{Fe} / \mathrm{H}]$ relationship.

A similar comparison of the results of the models with the observations can be made taking into consideration the masses

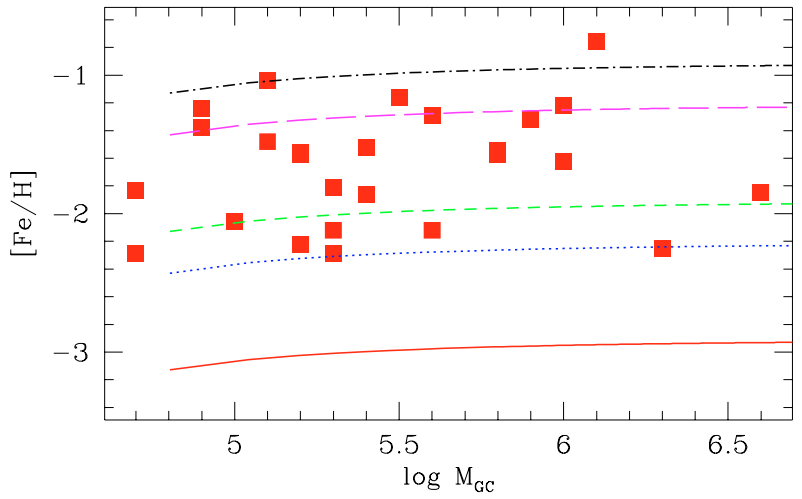

Fig. 10. Observed Mass-Metallicity relation for a selected sample of GCs (see Fig. 4) compared with model results. The mixing efficiency $\gamma$ is taken as 0.01 (solid line), 0.05 (dotted line), 0.1 (dashed line), 0.5 (long-dashed line) and 1 (dot-dashed line).

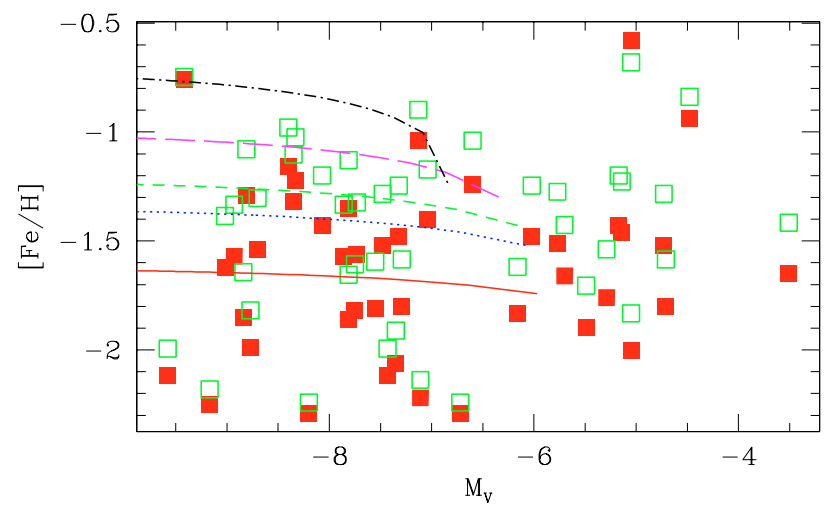

Fig. 11. Same as Fig. 9 but with varying lower mass $m_{1}$. This parameter ranges the values 0.1 (solid line), 0.5 (dotted line), 1 (dashed line), 3 (long-dashed line) and 10 (dot-dashed line).

of GCs instead of the magnitudes. In this case, we do not have to use Eq. (31) but we can directly compare the swept-up mass at the moment of the onset of the gravitational instabilities with the mass tabulated by Pryor \& Meylan (1993) (see Fig. 4). The results are plotted in Fig. 10. The conclusions we can draw from this plot are the same: a very low mixing efficiency can be ruled out and, in order to reproduce the GCs with the largest metallicities, we have to assume that more than $50 \%$ of the metals mix with the surrounding shell in a time-scale shorter than the time needed for the onset of gravitational instabilities.

\subsubsection{The magnitude-metallicity relation as a function of $m_{1}$}

Many authors believe that the IMF of primordial objects should have been top-heavy, owing to the fact that the CMB radiation keeps the gas warm enough to raise the Jeans mass. We therefore varied the lower limit of the IMF distribution $m_{1}$ in order to see what effect an IMF biased towards massive stars can have in the metallicity of GCs.

The magnitude-metallicity relation as a function of $m_{1}$ is shown in Fig. 11. In this set of models, the thermalization efficiency $\eta$ and the mixing efficiency $\gamma$ are set to be $10^{-4}$ and 0.5 , respectively. As we can see in this figure, an IMF strongly 


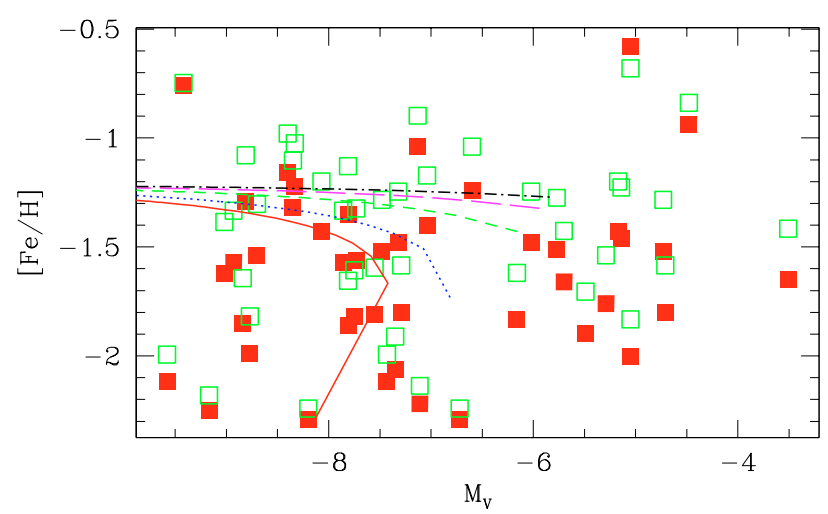

Fig. 12. Same as Fig. 9 but with varying thermalization efficiency $\eta$. This parameter ranges the values $5 \times 10^{-3}$ (solid line), $10^{-3}$ (dotted line), $10^{-4}$ (dashed line), $10^{-5}$ (long-dashed line) and $10^{-6}$ (dot-dashed line).

biased towards massive stars $\left(m_{1}=10\right)$ can justify the most metal-rich GC observed. If the IMF is so strongly top-heavy, mixing efficiencies larger that 0.5 cannot be allowed. This mixing efficiency is consistent with the value found by Recchi et al. (2001) in their simulations of IZw18. Owing to the fact that the selected sample of GCs is not perfectly coeval, small clusterto-cluster variations of $m_{1}$ are possible, explaining therefore, at least in part, the observed scatter.

Also for these models, $[\mathrm{Fe} / \mathrm{H}]$ does not vary so much with $M_{V}$. Only for models with large $m_{1}$ a correlation arises, in the sense that the faintest (and less massive) GCs are the most metal-rich. This is due to the fact that $t_{*}$ grows with $M_{6}$. At large $t, M_{Z}$ is almost independent on $t$ (see Fig. 6), whereas $M_{\text {sw }}$ grows linearly with $t$ (Eq. (21)). From Eq. (27) we can see that Z should decrease. This trend has been found also by P99. Statistical properties of a selected sample of old GCs (the socalled Old Halo GCs) seem to confirm this trend (Parmentier \& Gilmore 2001), although the scatter is huge.

\subsubsection{The magnitude-metallicity relation as a function of $\eta$}

The thermalization efficiency $\eta$ is the ratio of the mechanical energy of a single SN to the internal energy gained by the ISM after the explosion. As we have seen in Sect. 3.2, the value of $\eta$ can be estimated analytically (Eq. (9)) but, due to the large uncertainties in this evaluation, we decided to keep the thermalization efficiency as a free parameter. However, owing to the very large densities in the core of PGCs, this value should be very low. We therefore decided to span the parameter range $\left[5 \times 10^{-3}, 10^{-6}\right]$.

The magnitude-metallicity relation as a function of $\eta$ is shown in Fig. 12. In this set of models, the lower limit of the IMF distribution $m_{1}$ and the mixing efficiency $\gamma$ are set to be 1 and 0.5 , respectively. As we can see in this figure, below $\eta=10^{-4}$, the dependence of the metallicity with $\eta$ is very weak. Above this value, the dependence becomes stronger due to the non-linear dependence of $t_{*}$ (and consequently $M_{Z}\left(t_{*}\right)$ and $\left.M_{\mathrm{sw}}\left(t_{*}\right)\right)$ on $\eta$ (see Eq. (26)).

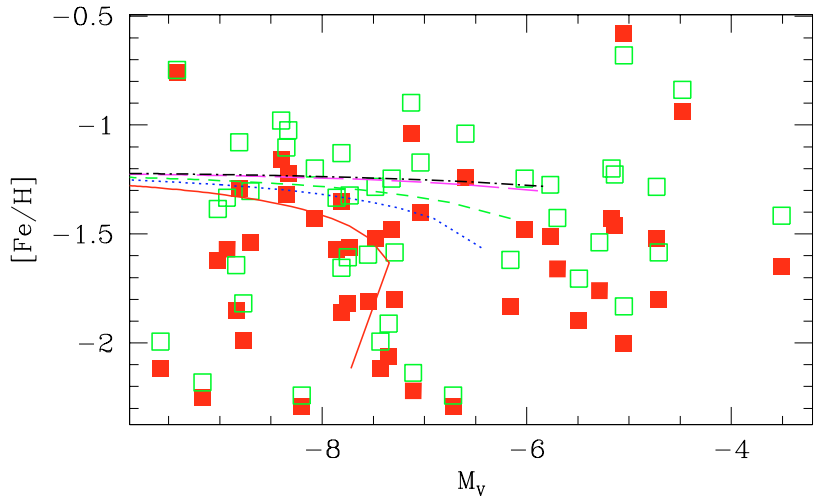

Fig. 13. Same as Fig. 9 but with varying external pressure $P_{4}$. This parameter ranges the values 1 (solid line), 5 (dotted line), 10 (dashed line), 50 (long-dashed line) and 100 (dot-dashed line).

\subsubsection{The magnitude-metallicity relation as a function of $P_{4}$}

So far we have analyzed the dependence of the $M_{V}$ vs. $[\mathrm{Fe} / \mathrm{H}]$ relation as a function of internal parameters of the PGC. Of course also external parameters play a role. In this subsection we analyze the evolution of the metallicity of GCs as a function of the external pressure $P_{4}$. This dependence is shown in Fig. 13.

The effect of the external pressure is similar to the effect of the thermalization efficiency $\eta$, in the sense that above $P_{4}=10$ this effect is negligible and the tracks lie close to each other. For low values of the external pressure, strongly non-linear effects on Eq. (26) create the knee observed for the model with $P_{4}=1$ (solid line) and $P_{4}=5$ (dotted line). Smaller values of $P_{4}$ violate the condition $3 c_{\mathrm{s}} v_{\mathrm{b}}<\sqrt{2} G \pi \rho_{0} r_{\mathrm{c}}^{2}$ (see Sect. 3.5) and cannot be taken into account in this simplified approach. As stated in Sect. 4.1, these low pressure environments are unlikely in protogalactic backgrounds.

\section{Conclusions}

We performed an analytical study of the evolution of a protoglobular cluster (PGC) assuming an initial burst of star formation occurring inside the core radius of the initial gaseous distribution. We followed the evolution of the shock wave formed after energy release from the first SNeII and we considered the possibility that gravitational instabilities may arise in the swept-up shell, leading to the formation of a second population of stars, with metallicity larger than zero, owing to the pollution from the ejecta of the first supernovae. Our results can be summarized as follows:

- Is it possible to calculate a lower mass of the PGC assuming that the onset of the gravitational instability able to produce the second generation of stars should occur before the shell reaches the edge of the PGC gaseous distribution. With reasonable choices of the parameters, this threshold mass is of the order of $10^{6} M_{\odot}$, in agreement with other similar studies (SW00, Brown et al. 1995).

- In order to produce a second stellar population with a metallicity in agreement with the one observed in the most 
metal-poor GCs, it is necessary to mix at least $5-10 \%$ of the metals produced in the central burst of star formation with the surrounding swept-up shell. The most metal-rich GCs are consistent with a very large mixing efficiency (larger than 0.5 ; i.e. more than $50 \%$ of the metals mix with the surrounding shell).

- The evolution of theoretical tracks in the $M_{V}$ vs. $[\mathrm{Fe} / \mathrm{H}]$ plot is almost constant. This is consistent with the fact that no obvious trend can be found in the observed $M_{V}$ vs. $[\mathrm{Fe} / \mathrm{H}]$ relationship. The scatter of the metallicity is therefore due mostly to scatter in the internal parameters of the cluster.

- A top-heavy IMF (with lower mass $m_{1}$ larger than $1 M_{\odot}$ ) seems to be required in order to explain the GCs with the largest metallicities.

- The huge spread in the metallicity observed in the sample of GCs can be explained, at least in part, with cluster-tocluster variations of the structural parameters $\gamma, \eta$ and $m_{1}$, as well as variations of the external pressure $P_{4}$.

Acknowledgements. We acknowledge an anonymous referee for suggestions and comments which improved the paper. S.R. acknowledges generous financial support from the Alexander von Humboldt Foundation and Deutsche Forschungsgemeinschaft (DFG) under grant HE 1487/28-1 and from the INAF (Italian National Institute for Astrophysics) - Osservatorio Astronomico di Trieste

\section{References}

Ashman, K. M., \& Zepf, S. E. 1998, Globular cluster systems (New York: Cambridge University Press).

Bate, M. R., Bonnell, I. A., \& Bromm, V. 2003, MNRAS, 339, 577

Beasley, M. A., Kawata, D., Pearce, F. R., Forbes, D. A., \& Gibson, B. K. 2003, ApJ, 596, L187

Bradamante, F., Matteucci, F., \& D'Ercole, A. 1998, A\&A, 337, 338

Brown, J. H., Burkert, A., \& Truran, J. W. 1995, ApJ, 440, 666

Carretta, E., \& Gratton, R. 1997, A\&AS, 121, 95

Cayrel, R. 1986, A\&A, 168, 81

Christlieb, N., Gustafsson, B., Korn, A., et al. 2004, ApJ, 603, 708

Carney, B. W., Laird, J. B., Latham, D. W., \& Aguilar, L. A. 1996, AJ, 112,668

Cioffi, D. F., McKee, C. F., \& Bertschinger, E. 1988, ApJ, 334, 252

Danziger, I. J. 1973, ApJ, 181, 641

Dekel, A., \& Silk, J. 1986, ApJ, 303, 39

Dopita, M. A., \& Smith, G. H. 1986, ApJ, 304, 283
Djorgovski, S. G., Gal, R. R., McCarthy, J. K., et al. 1997, ApJ, 474, L19

Fall, S. M., \& Rees, M. J. 1985, ApJ, 298, 28

Freeman, K. C., \& Rodgers, A. V. 1975, ApJ, 201, L71

Gonzalez, G., \& Wallerstein, G. 1998, AJ, 116, 765

Gratton, R. G., Bonifacio, P., Bragaglia, A., et al. 2001, A\&A, 369, 87

Harris, W. E. 1996, AJ, 112, 1487

Ikuta, C., \& Arimoto, N. 2000, A\&A, 358, 535

Koo, B.-C., \& McKee, C. F. 1992a, ApJ, 388, 93

Koo, B.-C., \& McKee, C. F. 1992b, ApJ, 388, 103

Kroupa, P. 2002, Science, 295, 82

Laird, J. B., Carney, B. W., \& Latham, D. W. 1993, in The globular clusters-galaxy connection, ed. G. H. Smith \& J. P. Brodie (San Francisco: ASP), 95

Langer, G. E., Fischer, D., Sneden, C., \& Bolte, M. 1998, AJ, 115, 685

Larsen, S. S., Brodie, J. P., Sarajedini, A., \& Huchra, J. P. 2002, AJ, 124,2615

Larson, R. B. 1974, MNRAS, 169, 229

Lehnert, W. D., Bell, R. A., \& Cohen, J. G. 1991, ApJ, 367, 514

Mateo, M. 1998, ARA\&A, 36, 435

Matteucci, F., Raiteri, C. M., Busso, M., Gallino, R., \& Gratton, R. 1993, A\&A, 272, 421

McCray, R., \& Kafatos, M. 1987, ApJ, 317, 190

Melioli, C., \& de Gouveia Dal Pino, E. M., 2004, A\&A, 424, 817

Morgan, S., \& Lake, G., 1989, ApJ, 339, 171

Murray, S. D., \& Lin, D. N. C. 1992, ApJ, 400, 265

Newberg, H. J., Yanny, B., Rockosi, C., et al. 2002, ApJ, 569, 245

Norris, J. E., \& Freeman, K. C. 1983, ApJ, 266, 130

Norris, J. E., \& Smith, G. H. 1981, in Astrophysical Parameters for Globular Clusters, ed. A. G. D. Philip \& D. S. Hayes (Schenectady: L. Davis Press), 109

Padovani, P., \& Matteucci, F. 1993, ApJ, 416, 26

Pancino, E., Pasquini, L., Hill, V., Ferraro, F. R., \& Bellazzini, M. 2002, ApJ, 568, L101

Parmentier, G., \& Gilmore, G. 2001, A\&A, 378, 97

Parmentier, G., Jehin, E., Magain, P., et al. 1999, A\&A, 352, 138 (P99)

Pryor, C., \& Meylan, G. 1993, in Structure and Dynamics of Globular Clusters, ed. S. G. Djorgovski and G. Meylan, ASP Conf. Ser., 50

Recchi, S., Matteucci, F., \& D'Ercole, A. 2001, MNRAS, 322, 800

Shustov, B. M., \& Wiebe, D. S. 2000, MNRAS, 319, 1047 (SW00)

Smith, G. H. 2000, PASP, 112, 12

Smith, V. V., Suntzeff, N. B., Cunha, C., et al. 2000, AJ, 119, 1239

Weaver, R., McCray, R., Castor, J., Shapiro, P., \& Moore, R. 1977, ApJ, 218, 377

Woosley, S. E. \& Weaver T. A. 1995, ApJS, 101, 181

Zinn, R. 1980, AJ, 85, 1468

Zinn, R., \& West, M. J. 1984, ApJS, 55, 45 